“( 2014 IEEE. Personal use of this material is permitted. Permission from IEEE must be obtained for all other uses, in any current or future media, including

reprinting/republishing this material for advertising or promotional purposes, creating new collective works, for resale or redistribution to servers or lists, or reuse of any copyrighted component of this work in other works." 


\title{
Novel Insights into the Impact of Graph Structure on SLAM
}

\author{
Kasra Khosoussi, Shoudong Huang and Gamini Dissanayake
}

\begin{abstract}
SLAM can be viewed as an estimation problem over graphs. It is well known that the topology of each dataset affects the quality of the corresponding optimal estimate. In this paper we present a formal analysis of the impact of graph structure on the reliability of the maximum likelihood estimator. In particular, we show that the number of spanning trees in the graph is closely related to the D-optimality criterion in experimental design. We also reveal that in a special class of linear-Gaussian estimation problems over graphs, the algebraic connectivity is related to the E-optimality design criterion. Furthermore, we explain how the average node degree of the graph is related to the ratio between the minimum of negative log-likelihood achievable and its value at the ground truth. These novel insights give us a deeper understanding of the SLAM problem. Finally we discuss two important applications of our analysis in active measurement selection and graph pruning. The results obtained from simulations and experiments on real data confirm our theoretical findings.
\end{abstract}

\section{INTRODUCTION}

\section{A. Motivation}

Simultaneous Localization and Mapping (SLAM) has been a central topic in robotics for more than 15 years [1]. In the state-of-the-art approaches SLAM is often formulated as a nonlinear least squares problem. Iterative methods such as Gauss-Newton (GN) are employed to find the minimizer of this nonlinear least squares problem which results in the maximum likelihood (ML) estimate under the assumption of Gaussian measurement noise. In recent years, through exploiting various structures and properties of SLAM such as sparsity of the information matrix, researchers have been focused on designing efficient algorithms for finding the ML estimate. As a result, a variety of solvers have been proposed in the literature, see e.g., [2], [3].

Despite the mature state of SLAM research, reliability is still a concern. For example, data association has always been and still is a challenge [4]. Also, all of the existing methods are subject to local minima [5]. In [6] authors show that for a special class of pose-graphs with spherical noise covariance matrices, the nonlinear least squares problem is equivalent to a one-dimensional problem for which there are at most 3 minima. Local optimization algorithms such as GN exhibit different convergence behaviours for different SLAM problems. Besides obvious factors such as measurement noise level, it has been shown in [7] that the graphical structure of the problem plays a role in the convergence of GN to the optimal solution. Note that the graphical structure of a dataset is affected by a variety of factors, including but

\footnotetext{
${ }^{\star}$ This work was supported by the Australian Research Council (ARC) Discovery Project DP 120102786.

The authors are with the Centre for Autonomous Systems (CAS), Faculty of Engineering and Information Technology, University of Technology Sydney, Australia. \{Kasra.Khosoussi, Shoudong.Huang, Gamini.Dissanayake\}@uts.edu.au
}

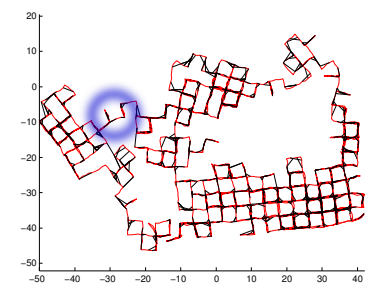

(a) Manhattan

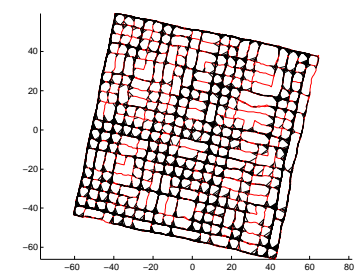

(b) City $10 \mathrm{~K}$
Fig. 1. ML estimates for two datasets with identical noise models.

not limited to the environment, chosen trajectory, available sensors and collected measurements.

It is obvious, but important to note that achieving the optimal estimate (in the ML sense) does not guarantee absolute accuracy. Normally the covariance matrix of the obtained solution is expected to describe the uncertainty associated to our estimate. But in some cases, errors due to linearization or even worse, converging to a local minimum, may prevent the computed covariance matrix from reflecting the true uncertainties. ${ }^{1}$ Moreover, updating or storing the (dense) covariance matrix in full SLAM quickly becomes intractable as the state vector grows.

Particular features of a given dataset determine the reliability of the associated optimal estimate. The impact of more direct factors such as the sensor noise and the observation model has been explored in the literature, see e.g., [8], [9]. It is also well known that structure of the corresponding graph (e.g., frequency of loop closures) has a great impact on the reliability of the ML estimate. As an (extreme) example consider a robot exploring the environment without closing any loops. In this case, the estimated poses obtained by performing dead-reckoning using the odometry data will be the ML estimate. Such an estimate clearly becomes more and more uncertain and inaccurate as the robot explores the environment. The graphical representation of such a dataset is a tree, which has the minimal connectivity among connected graphs. Intuitively speaking, maintaining a good "connectivity" in our graph means that our state variables are well-constrained, and therefore the ML estimate is less uncertain and more reliable. In order to illustrate this fact, we constructed two synthetic pose-graph datasets with identical noise models. Figure 1 shows the ML estimate for each dataset. Note that the accurate solution of City $10 \mathrm{~K}$ is mainly due to its better "connectivity". The bridges highlighted in Figure 1(a) are perfect examples of bad connectivity. ${ }^{2}$

\footnotetext{
${ }^{1}$ This case is referred to as inconsistency in the SLAM literature.

${ }^{2}$ In a connected graph, a bridge is an edge whose removal makes the graph disconnected.
} 
Therefore it is natural to ask questions like: what qualities of a given graph makes the corresponding ML estimate reliable? Is it possible to predict the highest achievable accuracy by just looking at the associated graph? The main purpose of this work is to address these questions.

\section{B. Contribution}

What we intuitively perceive as connectivity can be defined and measured in a number of different ways. Likewise, the uncertainty encoded in the corresponding covariance matrix can be measured (by a real number) in various ways depending on the application. The main contribution of this paper is to investigate the link between different definitions of connectivity, and reliability of the ML estimate. In particular we show that the number of spanning trees in the graph is closely related to the volume of confidence ellipsoids and the D-optimality design criterion [10]. Furthermore, we explain how the "diameter" of confidence ellipsoids and the E-optimality [10] design criterion are related to the algebraic connectivity [11] of the corresponding graph in a special case. We also explain how the average node degree is related to the ratio between the minimum of the negative log-likelihood function and its value at the ground truth; a phenomenon which was reported in [12].

In this work we introduce novel insights into the role of graph structure in the state-of-the-art formulation of SLAM. We believe that our analysis can set the stage for future works in data gathering and active SLAM scenarios, where the relevant definition of connectivity can be taken into account in the planning phase. Note that the importance of graph structure is not limited to SLAM. Connectivity also plays a major role in many estimation problems over graphs in e.g., sensor networks. See [13] and references therein for a number of real-world applications.

\section{Outline}

This paper is organized as follows. Section II provides some preliminaries and the problem formulation. Section III describes the main results relating the graph connectivity with the accuracy of ML estimate in SLAM. In Section IV, an application to quantify the value of each edge in a SLAM graph is provided. Finally a brief discussion and conclusion are given in Section V.

\section{PRELIMinaries}

\section{A. Problem Formulation}

Here we focus on the 2D pose-graph SLAM problem. A (simple) directed graph $\mathcal{G}=(\mathcal{V}, \mathcal{E})$ is a natural choice for representing this problem: each node $x_{i} \in \mathcal{V}$ denotes a robot pose, and each edge $e_{k} \triangleq\left(i_{k}, j_{k}\right) \in \mathcal{E}$ represents a relative measurement between two robot poses (odometry or loop-closure). Each $(i, j) \in \mathcal{E}$ in the graph corresponds to an observation from $x_{i}$ to $x_{j}$. Suppose $|\mathcal{V}|=n+1$ and $|\mathcal{E}|=m$. We denote the degree of vertex $x_{i}$ by $d_{i}$. In the absence of an informative prior and due to the relative nature of measurements we must define a global coordinates system and anchor one of the nodes to it. Without loss of generality, we can assume $x_{0}$ is the origin of our global coordinates system. The reduced incidence matrix of $\mathcal{G}$ is denoted by $A \in\{-1,0,1\}^{n \times m}$. $A$ is obtained by removing the row that corresponds to $x_{0}$ in the incidence matrix of $\mathcal{G}$. For the $k$ th edge $e_{k}=\left(i_{k}, j_{k}\right) \in \mathcal{E}$ we have $A_{i_{k}, k}=-1$ and $A_{j_{k}, k}=1$. The remaining elements of $A$ are all zero. The reduced Laplacian matrix of $\mathcal{G}$, after anchoring $x_{0}$, is defined as $L \triangleq A A^{\top}$. Note that $L$ is the principle submatrix of the Laplacian matrix $L_{0}$ after removing the row and column associated to $x_{0}$. It can be shown that $L$ is positive definite if and only if $\mathcal{G}$ is weakly connected, which is the case for a SLAM dataset (see Theorem 2). The reduced Laplacian matrix has a very simple structure: it can be written as $L=D-W$, where $D \triangleq \operatorname{diag}\left(d_{1}, \ldots, d_{n}\right)$, and $W$ is the adjacency matrix of graph after removing the row and column corresponding to $x_{0}$.

The corresponding state vector is usually defined as $x_{\text {old }} \triangleq\left[x_{1}^{\top}, \ldots, x_{n}^{\top}\right]^{\top}$. Similar to [7], we permute this standard state vector and define ours as $x \triangleq\left[p^{\top}, \theta^{\top}\right]^{\top}$. Here $p \in \mathbb{R}^{2 n}$ is the vector of $x$ and $y$ coordinates of robot poses, and $\theta \in[-\pi, \pi)^{n}$ is the vector of robot orientations. Let $z_{i j}$ be the observation from pose $i$ to pose $j$. We assume each observation is corrupted by an independently drawn additive Gaussian noise, ${ }^{3}$

$$
z_{i j}=h_{i j}\left(x_{i}, x_{j}\right)+w_{i j}
$$

where $w_{i j} \sim \mathcal{N}\left(0, \Sigma_{i j}\right)$. The measurement function $h_{i j}(\cdot, \cdot)$ is defined as

$$
h_{i j}\left(x_{i}, x_{j}\right)=\left[\begin{array}{l}
\delta p_{i j} \\
\delta \theta_{i j}
\end{array}\right]=\left[\begin{array}{c}
R\left(\theta_{i}\right)^{\top}\left(p_{j}-p_{i}\right) \\
\operatorname{wrap}\left(\theta_{j}-\theta_{i}\right)
\end{array}\right],
$$

in which $p_{i}$ denotes the position of the $i$ th robot pose, $R\left(\theta_{i}\right)$ is the rotation matrix corresponding to $\theta_{i}$, and wrap : $\mathbb{R} \rightarrow[-\pi, \pi)$ is the function that maps its argument to the equivalent angle in $[-\pi, \pi)$. Let $z_{p}$ and $z_{\theta}$ denote the stacked vector of $\delta p_{i j}$ and $\delta \theta_{i j}$ measurements, respectively. As in [7], in order to replace wrap $\left(\theta_{j}-\theta_{i}\right)$ with $\theta_{j}-\theta_{i}$ we need to assume that correct regularization terms have been computed for the measured $\delta \theta_{i j}$ [14]. The ML estimate $\hat{x}$ is obtained by minimizing the negative log-likelihood function $\hat{x}=\arg \min r^{\top} \Sigma^{-1} r$, where for pose-graphs

$$
r \triangleq\left[\begin{array}{c}
r_{p} \\
r_{\theta}
\end{array}\right] \triangleq\left[\begin{array}{c}
z_{p}-R^{\top} A_{2}^{\top} p \\
z_{\theta}-A^{\top} \theta
\end{array}\right]
$$

Here $\Sigma$ is obtained by permuting the rows and columns of the covariance matrix of stacked noise vector, $A_{2} \triangleq A \otimes I_{2}{ }^{4}$ and $R$ is defined as

$$
R \triangleq \operatorname{diag}\left(R\left(\theta_{k_{1}}\right), \ldots, R\left(\theta_{k_{m}}\right)\right)
$$

in which $k_{i}$ is the index of robot pose generating the $i$ th measurement. The Jacobian matrix of $r$ is given by

$$
J \triangleq\left[\begin{array}{ll}
\frac{\partial r_{p}}{\partial p} & \frac{\partial r_{p}}{\partial \theta} \\
\frac{\partial r_{\theta}}{\partial p} & \frac{\partial r_{\theta}}{\partial \theta}
\end{array}\right]=\left[\begin{array}{cc}
-R^{\top} A_{2}^{\top} & -\bar{R} \Delta \\
0 & -A^{\top}
\end{array}\right],
$$

\footnotetext{
${ }^{3}$ For simplicity, we use the same notation for random variables and their realization.

${ }^{4} \otimes$ and $I_{k}$ denote the Kronecker product and the identity matrix of size $k$, respectively.
} 
where

$$
\bar{R} \triangleq \operatorname{diag}\left(R\left(\frac{3 \pi}{2}-\theta_{k_{1}}\right), \ldots, R\left(\frac{3 \pi}{2}-\theta_{k_{m}}\right)\right),
$$

and for each $e_{k}=\left(i_{k}, j_{k}\right) \in \mathcal{E}$, there is a 2-by-1 block in $\Delta \in \mathbb{R}^{2 m \times n}$ that contains

$$
\left[\begin{array}{c}
\Delta_{2 k-1, i_{k}} \\
\Delta_{2 k, i_{k}}
\end{array}\right]=p_{j_{k}}-p_{i_{k}}
$$

The remaining elements in $\Delta$ are all zero. Finally it is worth noting that $\Delta^{\top} \Delta$ is a diagonal matrix: $\left(\Delta^{\top} \Delta\right)_{i, i}$ is equal to the sum of squared distances between the $i$ th robot pose, and all of the nodes observed by it [7].

Assumption 1. For each measurement $(i, j) \in \mathcal{E}$ we have $\Sigma_{i j}=\operatorname{diag}\left(\sigma_{p}^{2} I_{2}, \sigma_{\theta}^{2}\right)$.

\section{B. The Cramér-Rao Lower Bound}

In this section we briefly review the Cramér-Rao lower bound (see e.g., [15]). Here we treat $x$ as a vector of (deterministic ${ }^{5}$ ) unknown parameters. $\hat{x}$ is called an unbiased estimator if $\mathbb{E}_{z}[\hat{x}]=x$ (expectation is over $z$ and with respect to $p(z ; x)$ ). The uncertainty associated with the ML estimate is encoded in the estimation error covariance matrix.

Theorem 1. [Cramér-Rao Lower Bound (CRLB)] Under some regularity conditions [15], the covariance matrix of any unbiased estimator of $x$, such as $\hat{x}$, satisfies $\mathbb{C o v}[\hat{x}] \succeq \mathcal{I}^{-1}(x),{ }^{6}$ where $\mathcal{I}(x)$ is the Fisher information matrix (FIM),

$$
\mathcal{I}(x) \triangleq \mathbb{E}_{z}\left[\frac{\partial}{\partial x} \log p(z ; x) \frac{\partial^{\top}}{\partial x} \log p(z ; x)\right] .
$$

Here the expectation is over $z$ and with respect to $p(z ; x)$. Note that FIM depends only on the true value of $x$ and $p(z ; x)$, and does not depend on any particular realization of $z$. An unbiased estimator that achieves CRLB is called efficient.

Remark 1. For our nonlinear-Gaussian model (1) FIM is given by $\mathcal{I}(x)=J^{\top} \Sigma^{-1} J$ in which $J$ is the Jacobian matrix of $r$ (see e.g., [15]).

Definition 1. We use the term "compass-SLAM" to refer to the SLAM problem when the robot heading is known (e.g., using a compass).

Definition 2. We use the term "linear-SN" (sensor network) to refer to the state estimation problem over sensor networks when $z=A_{d}^{\top} x+w$, in which $w$ is the stacked noise vector and $A_{d} \triangleq A \otimes I_{d}$. In particular, here we consider the case with $d=3$ in which each measurement is simply the difference between the position and orientation of two nodes, corrupted by zero-mean Gaussian noise. However note that our results can be straightforwardly generalized for

\footnotetext{
${ }^{5}$ This is the most common approach to SLAM, as in most of real scenarios no a priori information is available about the trajectory and/or map. In a Bayesian approach, Gaussian prior over poses/features can be incorporated into the least squares formulation in order to obtain the maximum $a$ posteriori (MAP) estimate.

${ }^{6} A \succeq B$ means $A-B$ is positive semidefinite. As a consequence we have $\overline{A_{i, i}} \geq B_{i, i}$. In other words, the diagonal elements of CRLB are lower bounds on the variance of any unbiased estimator for each parameter.
}

any integer $d \geq 1$. See [13] and references therein for a number of real-world applications.

Remark 2. In compass-SLAM, $J=-R^{\top} A_{2}^{\top}$. Note that here $R$ is a constant matrix computed from the true robot orientations. Likewise, the measurement function in linear-SN is simply the difference between the corresponding components of two nodes. Let $L_{2} \triangleq L \otimes I_{2}=A_{2} A_{2}^{\top}$. Then it is straightforward to show that

$$
\mathcal{I}(x)= \begin{cases}\frac{1}{\sigma_{p}^{2}} L_{2} & \text { for compass-SLAM, } \\ \operatorname{diag}\left(\frac{1}{\sigma_{p}^{2}} L_{2}, \frac{1}{\sigma_{\theta}^{2}} L\right) & \text { for linear-SN. }\end{cases}
$$

From (9) it is clear how graph structure is strongly related to FIM through $L$. It is important to note that the maximum likelihood estimator (MLE) is unbiased and efficient in both compass-SLAM and linear-SN problems.

\section{GRAPH CONNECTIVITY IN SLAM}

Although our results cover both the feature-based and pose-graph problems, here we mainly focus on the posegraph formulation in order to simplify our notation. In the following sections we first look at a heuristic measure of reliability defined in [12]. Then we focus on the estimation error covariance matrix of MLE. Let us use $x^{\circ}$ to denote the true trajectory. Note that $A^{\top}$ and $A_{2}^{\top}$ are full column rank and $R$ is invertible. Therefore $J$ is full column rank regardless of the value of $x$. Hence our focus will be on $\mathcal{I}^{-1}(x)=\left(J^{\top} \Sigma^{-1} J\right)^{-1}$. Note that:

1) $\mathcal{I}(x)$ evaluated at the ground truth $x=x^{\circ}$, is the FIM. Therefore $\mathcal{I}^{-1}(x)$ evaluated at $x^{\circ}$ gives CRLB, i.e., an upper bound on the achievable "accuracy" for unbiased estimators.

2) The covariance matrix of MLE is usually approximated by evaluating $\mathcal{I}^{-1}(x)$ at the ML estimate $\hat{x}$, known as the observed Fisher information matrix. Note that this value, unlike FIM, depends on the given realization of observations.

Therefore, assuming the back-end has converged to the ML estimate, $\mathcal{I}^{-1}(\hat{x})$ tells us about the accuracy of MLE, while $\mathcal{I}^{-1}\left(x^{\circ}\right)$ gives an upper bound on the achievable accuracy by any unbiased estimator. Note that MLE is asymptotically unbiased and efficient [15]. Consequently we look at the relation between FIM and different definitions of connectivity.

\section{A. Ratio of Costs $\Leftrightarrow$ Average Node Degree}

Olson and Kaess [12] briefly discussed the role of graph structure in SLAM. They looked at the ratio between the minimum of ML objective function $f^{\star}$, and its value at the ground truth $f^{\circ}$. For this ratio, $\gamma \triangleq f^{\star} / f^{\circ}$, we have: (i) $0 \leq \gamma \leq 1$, and (ii) $\gamma \rightarrow 1$ as $\hat{x} \rightarrow x^{\circ}$. Therefore $\gamma$ can be used as a heuristic measure to reflect the accuracy of the ML estimate. Through Monte Carlo simulations, authors in [12] empirically observed that as the average node degree $\bar{d}=2 \mathrm{~m} / \mathrm{n}$ in the graph increases, the average of $\gamma$ approaches to 1 (see Figure 5 in [12]). Olson and Kaess interpret $\gamma$ as a coarse measure of overfitting.

Here we first give a theoretical justification for this empirical observation. Please refer to Section V for a discussion 


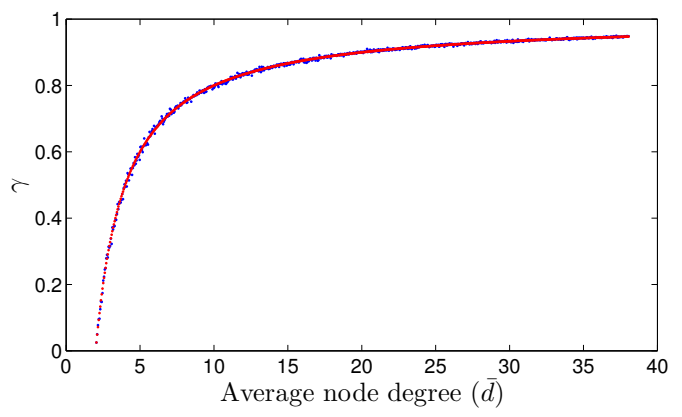

Fig. 2. Average of $\gamma$ in 50 Monte Carlo simulations for different average node degrees (blue). Estimated value of $\mathbb{E}[\gamma] \approx 1-\frac{2}{d}$ (red).

of the limitations of $\bar{d}$ and $\gamma$ as measures of "connectivity" and "accuracy", respectively. For the observation model described in (1), the value of objective function at the ground truth $x^{\circ}$ is given by $f^{\circ}=w^{\top} \Sigma^{-1} w$ in which $w$ is the stacked noise vector. In pose-graphs, $f^{\circ}$ is distributed according to a $\chi_{\nu_{1}}^{2}$ distribution with $\nu_{1}=3 m$ degrees of freedom. Furthermore, it is well known that for linear measurement functions, $f^{\star}$ is also distributed according to a $\chi_{\nu_{2}}^{2}$ distribution with $\nu_{2}=3(m-n)$ degrees of freedom [15]. For sufficiently large number of observations, it is common to extend this result to nonlinear measurement functions by linearizing $h_{i j}(\cdot, \cdot)$ at $x^{\circ}$ [16]. Therefore, for sufficiently large $m$ we have

$$
\mathbb{E}[\gamma] \approx \frac{\mathbb{E}\left[f^{\star}\right]}{\mathbb{E}\left[f^{\circ}\right]} \approx \frac{\nu_{2}}{\nu_{1}}=1-\frac{2}{\bar{d}} .
$$

Equation (10) clearly shows how $\gamma$, as a measure of accuracy of the ML solution in SLAM, is related to the average node degree $\bar{d}$, as the simplest measure of connectivity in graphs.

Results: The blue points in Figure 2 correspond to the average of $\gamma$ in a Monte Carlo simulation over different SLAM problems with different average node degree (similar to Figure 5 in [12]). For each random graph we generated 50 iid samples for measurement noise and then computed the average of $\gamma$ over those samples. The red points are obtained using the approximate expected value of $\gamma$ derived in (10). Figure 2 clearly indicates that (10) gives a good estimate for $\mathbb{E}[\gamma]$, and confirms the empirical observation in [12].

\section{B. Volume of Confidence Ellipsoid $\Leftrightarrow$ Number of Spanning Trees}

Determinant of the covariance matrix is a well-studied scalar measure of the quality of an estimate. Geometrically, for Gaussian random variables, determinant of the covariance matrix is proportional to the volume of confidence ellipsoids. Minimizing the determinant of the covariance matrix of MLE is known as the D-optimality criterion [10]. D-optimality is a popular criterion in active SLAM [17], especially due to its information-theoretic meaning for Gaussian random variables [18].

\footnotetext{
${ }^{7}$ This simple estimate is obtained using a first-order Taylor expansion of $\gamma$ at $f^{\star}=\mathbb{E}\left[f^{\star}\right]$ and $f^{\circ}=\mathbb{E}\left[f^{\circ}\right]$. Note that in general, approximating the expected value of ratio by the ratio of expected values does not result in a reasonable estimate.
}

Number of spanning trees in a graph, $t$, is a welldefined measure of connectivity. Among connected graphs with $n$ vertices, $\mathrm{t}$ is minimum in trees $(\mathrm{t}=1)$, while the complete graph has the maximum number of spanning trees ( $\mathrm{t}=n^{n-2}$, according to Cayley's formula). Adding a new edge to a connected graph always increases the number of spanning trees. However, the number of spanning trees in the new graph depends on the current graph structure as well as the selected edge.

Theorem 2. [Matrix-Tree Theorem [19]] The number of spanning trees of graph $\mathcal{G}$, i.e., $\mathrm{t}(\mathcal{G})$, is given by $\mathrm{t}(\mathcal{G})=\operatorname{det}\left(L_{\mathcal{G}}\right)$. Here $L_{\mathcal{G}}$ is the reduced Laplacian matrix of $\mathcal{G}$ after anchoring an arbitrary vertex.

From Theorem 2 and Remark 2 it readily follows:

Corollary 1. Let $t$ be the number of spanning trees in a given graph. Then the determinant of CRLB (or, equivalently, $\operatorname{det} \operatorname{Cov}[\hat{x}])$ in compass-SLAM and linear-SN is given by

$$
\operatorname{det}(\operatorname{Cov}[\hat{x}])= \begin{cases}\sigma_{p}^{4 n} \mathrm{t}^{-2} & \text { for compass-SLAM, } \\ \sigma_{p}^{4 n} \sigma_{\theta}^{2 n} \mathrm{t}^{-3} & \text { for linear-SN. }\end{cases}
$$

Remark 3. According to Corollary 1 , for a fixed noise model and number of poses, in compass-SLAM and linear-SN problems, the ratio of number of spanning trees in two graphs (e.g., datasets or planned trajectories) determines their relative D-optimality performance. Therefore the D-optimal "design" is the one with the maximum number of spanning trees possible.

Now let us consider the nonlinear SLAM problem defined in Section II. We start by computing a closed-from expression for $\mathcal{I}(x)=J^{\top} \Sigma^{-1} J$ in pose-graphs. Using (5) we have $\mathcal{I}(x) \triangleq\left[\begin{array}{ll}\mathcal{I}_{11} & \mathcal{I}_{12} \\ \mathcal{I}_{12}^{\top} & \mathcal{I}_{22}\end{array}\right]=\left[\begin{array}{cc}\sigma_{p}^{-2} L_{2} & \sigma_{p}^{-2} A_{2} \Gamma \Delta \\ \sigma_{p}^{-2} \Delta^{\top} \Gamma^{\top} A_{2}^{\top} & \sigma_{p}^{-2} L+\sigma_{\theta}^{-2} \Delta^{\top} \Delta\end{array}\right]$,

where $\Gamma$ is the following block-diagonal matrix

$$
\Gamma \triangleq I_{m} \otimes\left[\begin{array}{rr}
0 & 1 \\
-1 & 0
\end{array}\right]
$$

Remark 4. For feature-based SLAM, instead of $L$ in $\mathcal{I}_{22}$ we have $B_{n_{p}} B_{n_{p}}^{\top}$ in which $B_{n_{p}}$ is the reduced incidence matrix of odometry subgraph. Note that $B_{n_{p}}$ is uniquely determined by the number of poses $n_{p}$.

In (12) we obtained a closed-form expression for $\mathcal{I}(x)$. But recall that we are interested in $\mathcal{I}^{-1}(x)$, and not in $\mathcal{I}(x)$ itself. First note that $\operatorname{det} \mathcal{I}^{-1}(x)=1 / \operatorname{det} \mathcal{I}(x)$. Therefore we can focus on $\operatorname{det} \mathcal{I}(x)$. Let us define $\overline{\mathcal{I}}(x) \triangleq \sigma_{p}^{2} \mathcal{I}(x)$. Let $S_{11}$ be the Schur complement of the top-left block in $\overline{\mathcal{I}}(x)$.

$$
\begin{aligned}
S_{11} & \triangleq \overline{\mathcal{I}}_{22}-\overline{\mathcal{I}}_{12}^{\top} \overline{\mathcal{I}}_{11}^{-1} \overline{\mathcal{I}}_{12} \\
& =L+\alpha^{2} \Delta^{\top} \Delta-\Delta^{\top} \Gamma^{\top} A_{2}^{\top} L_{2}^{-1} A_{2} \Gamma \Delta,
\end{aligned}
$$

in which $\alpha^{2} \triangleq \sigma_{p}^{2} / \sigma_{\theta}^{2}$. From the Schur determinant formula [20, Chapter 6.2] we have $\operatorname{det} \overline{\mathcal{I}}(x)=\operatorname{det}\left(\overline{\mathcal{I}}_{11}\right) \operatorname{det}\left(S_{11}\right)$. Here $\operatorname{det}\left(\overline{\mathcal{I}}_{11}\right)$ can be computed in closed-form.

$$
\operatorname{det}\left(\overline{\mathcal{I}}_{11}\right)=\operatorname{det}\left(L_{2}\right)=\operatorname{det}(L)^{2} \underset{\text { Theorem } 2}{=} \mathrm{t}^{2},
$$


where $t$ is the number of spanning trees of the graph. Now we only need to compute $\operatorname{det}\left(S_{11}\right)$. Recall that $L \succ 0$. Let $L^{\frac{1}{2}}$ be the symmetric positive definite square root of $L$, i.e., $L=L^{\frac{1}{2}} L^{\frac{1}{2}}$. Now $\operatorname{det}\left(S_{11}\right)$ can be rewritten as

$$
\begin{aligned}
\operatorname{det}\left(S_{11}\right) & =\operatorname{det}\left(L+\Delta^{\top}\left(\alpha^{2} I-\Gamma^{\top} A_{2}^{\top} L_{2}^{-1} A_{2} \Gamma\right) \Delta\right) \\
& =\operatorname{det}\left(L^{\frac{1}{2}}[I+T] L^{\frac{1}{2}}\right) \\
& =\operatorname{tdet}(I+T)
\end{aligned}
$$

where

$$
T \triangleq L^{-\frac{1}{2}} \Delta^{\top}\left(\alpha^{2} I-\Gamma^{\top} A_{2}^{\top} L_{2}^{-1} A_{2} \Gamma\right) \Delta L^{-\frac{1}{2}} .
$$

Finally according to (15) and (16) we have:

$$
\operatorname{det} \overline{\mathcal{I}}(x)=\mathrm{t}^{3} \operatorname{det}(I+T),
$$

or, equivalently,

$$
\operatorname{det} \mathcal{I}(x)=\sigma_{p}^{-6 n} \mathrm{t}^{3} \operatorname{det}(I+T) .
$$

Results: We performed a series of realistic pose-graph SLAM simulations (for both diagonal and correlated noise covariance matrices ${ }^{8}$ ) and experiments on real data (Intel Research Lab dataset) in order to understand the behaviour of $\operatorname{det}(I+T)$. The simulated trajectory was a circle. By selecting edges randomly, we created a large set of random graphs with different structures. According to our observations, for a large enough $\alpha$ (i.e., sufficiently small orientation noise $\sigma_{\theta}^{2}$ ), $\operatorname{det}(I+T)$ becomes close to a constant number, independent of the graph structure. Based on this observation, for a given noise model, we can estimate the determinant of FIM according to $\operatorname{det}(\mathcal{I}(x)) \approx \eta t^{3}$ in which $\eta$ is a constant number. Note that $t=1$ for any spanning tree. Therefore we can estimate $\eta$ by computing the determinant of FIM for a spanning tree such as the odometry subgraph. Taking the logarithm of both sides we get

$$
\log \operatorname{det}(\mathcal{I}(x)) \approx 3 \log (\mathrm{t})+\log (\eta) .
$$

Figure 3 shows the results. Similar results were obtained for part of the Manhattan simulation, ${ }^{9}$ but are not reported here due to space limitation. We also performed the same experiment on the first 180 poses of the Intel Research Lab dataset. ${ }^{10}$ We created a large set of random (sub)graphs by randomly selecting different combinations of loop-closure edges, and then evaluated the (observed) FIM at the ML estimate obtained by using the whole dataset. The results can be seen in Figure 4. Our results indicate that for a fixed number of poses, a fixed measurement model and a small enough $\sigma_{\theta}$, similar to compass-SLAM and linear-SN, number of spanning trees determines the D-optimality performance of MLE.

Remark 5. It is interesting to see that one can accurately estimate the determinant of FIM when the orientation measurements are sufficiently accurate. Nevertheless, it is of

\footnotetext{
${ }^{8}$ Correlation between the components of each measurement.

${ }^{9}$ Due to the numerical sensitivity of the determinant, we were limited to the first 200-300 poses of the Manhattan dataset.

${ }^{10}$ The Intel Research Lab dataset in g2o format can be found at http://openslam.org/g2o.html.
}

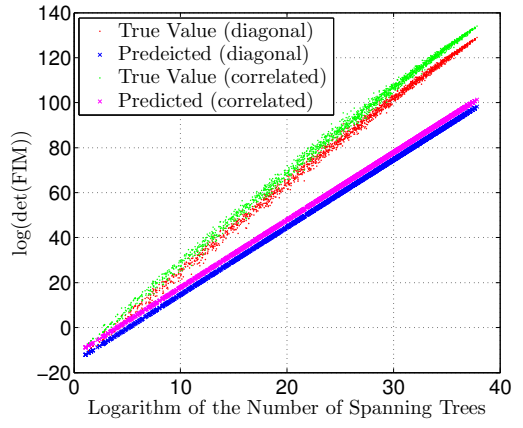

(a) $\sigma_{\theta}=0.15$ radian

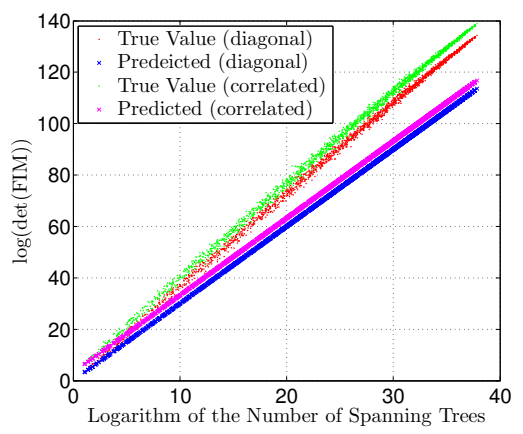

(b) $\sigma_{\theta}=0.1$ radian

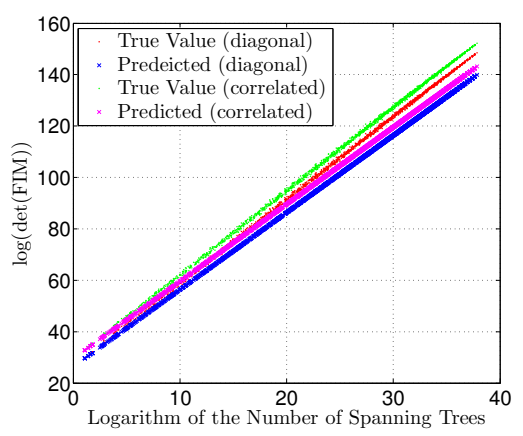

(c) $\sigma_{\theta}=0.05$ radian

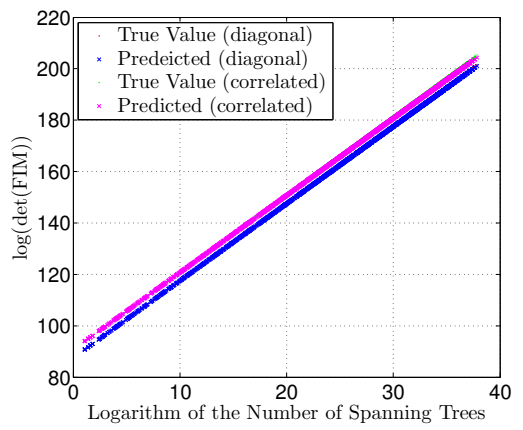

(d) $\sigma_{\theta}=0.01$ radian (The true and predicted values are on top of each other.)

Fig. 3. Results of Pose-Graph SLAM Simulation with $n=20$ poses and $\sigma_{p}=0.1$ meter. The vertical axis is $\log \operatorname{det} \overline{\mathcal{I}}(x)$, and the horizontal axis is $\log (\mathrm{t})$. Each green point corresponds to a random graph with correlated noise components. Each red point corresponds to a random graph with diagonal noise covariance matrix. Magenta and blue markers correspond to the predicted values according to (20). They both lie on a line with slope 3. The offset of the line is $\log \operatorname{det}(I+T)$ which is obtained from the odometry subgraph. 


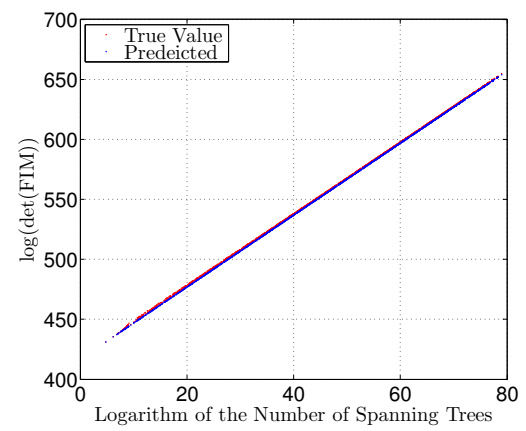

Fig. 4. Intel Research Lab Dataset (first 180 poses). $\sigma_{p}=0.04$ meter and $\sigma_{\theta}=0.01$ radian. The vertical axis is $\log \operatorname{det} \overline{\mathcal{I}}(x)$. Predicted values are obtained using (20). The offset is computed using the odometry subgraph.

utmost importance to note that according to Figure 3, even when $\alpha$ is not large enough and therefore our prediction (20) is not accurate, there is still a substantial positive correlation between $\log (t)$ and $\log \operatorname{det} \overline{\mathcal{I}}(x)$. This suggests that even for larger orientation measurement noises, the number of spanning trees is still a reasonable measure of connectivity in terms of the D-optimality criterion.

\section{Diameter of Confidence Ellipsoid $\Leftrightarrow$ Algebraic Connec- tivity}

According to Remark 2, the estimation error covariance matrix in linear-SN and compass-SLAM problems is given by CRLB. We also showed that in these cases, CRLB is closely related to the inverse of the reduced Laplacian matrix. Therefore, using (9) we can show that the largest eigenvalue of CRLB in linear-SN and compass-SLAM is proportional to $\lambda_{\min }(L)^{-1}$, in which $\lambda_{\min }(L)$ is the smallest eigenvalue of the reduced Laplacian matrix. Recall that $L$ is a principle submatrix of the Laplacian matrix $L_{0}$ obtained by deleting the row and column corresponding to $x_{0}$. In connected graphs, the Laplacian matrix $L_{0}$ is positive semidefinite [19]. It can be easily verified that the smallest eigenvalue of $L_{0}$ is zero. In fact, the corresponding eigenvector is the vector of ones. Let $\lambda_{2}$ be the second-smallest eigenvalue of $L_{0}$, known as the algebraic connectivity of graph [11]. As its name suggests, algebraic connectivity is a well-studied measure of network connectivity and robustness and has been used in many applications (see e.g., [21] and references therein).

Corollary 2. Theorem 9.1.1 in [19] (Cauchy's interlace theorem) implies that the eigenvalues of $L$ interlace the eigenvalues of $L_{0}$. In particular we have $0<\lambda_{\min }(L) \leq \lambda_{2}$ and therefore, $\lambda_{\min }(L)^{-1} \geq \lambda_{2}^{-1}$. Consequently in linear-SN and compass-SLAM problems we have

$$
\lambda_{\max }(\mathbb{C o v}[\hat{x}]) \geq \kappa \lambda_{2}^{-1},
$$

where according to (9) $\kappa$ is given by

$$
\kappa= \begin{cases}\sigma_{p}^{2} & \text { for compass-SLAM, } \\ \max \left\{\sigma_{p}^{2}, \sigma_{\theta}^{2}\right\} & \text { for linear-SN. }\end{cases}
$$

Note that $\lambda_{\max }(\operatorname{Cov}[\hat{x}])$ is the worst case estimation error variance over all (normalized) directions [22]. Geometrically, its square root is proportional to the "diameter" of the confidence ellipsoid for Gaussian random variables. The conclusion here is that for a given noise model in linear-SN and compass-SLAM, a necessary condition for having a "small" worst case error variance is to maintain a sufficiently large algebraic connectivity in the corresponding graph.

\section{Applications: Measurement Selection And EDGE PRUNING}

Here we look at two relevant applications in SLAM: active measurement selection and graph pruning. According to the results presented in the previous section, we can measure the importance of each edge in a given graph, based on its influence on the number of spanning trees. It is obvious that removing an edge reduces the number of spanning trees, while adding an edge always increases $t$. Therefore we can define the followings:

- The least valuable edge in a given graph is the one whose deletion causes the smallest decrease in $t$.

- The most valuable edge is the one that adding it to the given graph increases $t$ the most.

Hence we can prune the graph by removing less valuable edges, or we can select the most valuable measurement from a set of available candidates, in order to maintain the sparsity of information matrix without putting the reliability (in terms of D-optimality criterion) of MLE at risk.

Suppose $\mathcal{G}^{\prime}$ is the graph obtained by adding a new edge $e^{\prime} \triangleq(i, j)$ to $\mathcal{G}$. Then the reduced incidence matrix of $\mathcal{G}^{\prime}$ can be written as $A^{\prime}=\left(\begin{array}{ll}A & a^{i j}\end{array}\right)$, in which the only nonzero elements of vector $a^{i j}$ are $a_{i}^{i j}=-1$ and $a_{j}^{i j}=1$. The reduced Laplacian matrix of $\mathcal{G}^{\prime}$ can be written as

$$
L^{\prime} \triangleq A^{\prime} A^{\prime \top}=L+a^{i j} a^{i j^{\top}} .
$$

In this case, the ratio of number of spanning trees is given in Lemma 1.

Lemma 1. Let $t$ and $t^{\prime}$ be the number of spanning trees in $\mathcal{G}$ and $\mathcal{G}^{\prime}$, respectively. Then we have

$$
\begin{aligned}
\mathrm{t}^{\prime} / \mathrm{t} & =1+a^{i j^{\top}} L^{-1} a^{i j} \\
& =1+\left(L^{-1}\right)_{i, i}+\left(L^{-1}\right)_{j, j}-2\left(L^{-1}\right)_{i, j} .
\end{aligned}
$$

Proof. See Appendix I for the proof.

\section{A. Active Measurement Selection}

According to Lemma 1, given a graph and a set of new candidate measurements $\mathcal{C}^{+}$, the optimal choice with respect to the number of spanning trees is given by

$$
\left(i^{\star}, j^{\star}\right)=\underset{(i, j) \in \mathcal{C}^{+}}{\arg \max }\left(L^{-1}\right)_{i, i}+\left(L^{-1}\right)_{j, j}-2\left(L^{-1}\right)_{i, j} .
$$

\section{B. Graph Pruning}

Suppose we want to prune the least valuable measurement (with respect to the number of spanning trees) from a given graph $\mathcal{G}=(\mathcal{V}, \mathcal{E})$. Let $L$ be the reduced Laplacian matrix of $\mathcal{G}$. Then according to Lemma 1 , the optimal choice for pruning a single edge from a given set of candidates $\mathcal{C}^{-} \in \mathcal{E}$ is given by

$$
\left(i^{\star}, j^{\star}\right)=\underset{(i, j) \in \mathcal{C}^{-}}{\arg \min }\left(L^{-1}\right)_{i, i}+\left(L^{-1}\right)_{j, j}-2\left(L^{-1}\right)_{i, j} .
$$


Remark 6. It is interesting to see that for linear-SN and compass-SLAM problems, the objective function in (26) and (27) is closely related to our uncertainty about $\hat{x}_{j}-\hat{x}_{i}$, i.e., the relative state of $x_{j}$ and $x_{i}$. In other words, for linear-SN and compass-SLAM, (26) and (27) suggest to add/remove the edge between two vertices only if we have the largest/smallest uncertainty in predicting the value of that edge. See also [23].

\section{The Cost of Decision Making}

The reduced Laplacian matrix $L=D-W$ is sparse in realistic SLAM scenarios, while in general $L^{-1}$ is dense. Therefore it is obvious that computing $L^{-1}$ does not lead to a scalable strategy. The objective function in (26) and (27) is given by $f(i, j) \triangleq a^{i j}{ }^{\top} L^{-1} a^{i j}$. We can evaluate $f(i, j)$ at any $(i, j) \in \mathcal{V} \times \mathcal{V}$ efficiently using the following procedure.

1) Solve the sparse linear system $L b=a^{i j}$ using the Cholesky decomposition of $P L P^{\top}$ in which $P$ is a fill-reducing permutation [24].

2) Compute $f(i, j)=b_{j}-b_{i}$ in which $b_{k}$ is the $k$ th element of vector $b$.

It is important to note that the Cholesky decomposition needs to be calculated only once per graph, i.e., only for the first $(i, j)$ in our candidate set. For the rest of candidates we only need to perform sparse forward and back substitution which can be done in parallel for different $(i, j)$.

Now note that $\left|\mathcal{C}^{+}\right| \leq n(n+1) / 2-m$ and $\left|\mathcal{C}^{-}\right| \leq m$. Fortunately, the number of available new measurements $\left|\mathcal{C}^{+}\right|$ is small in practice because of the limited sensor range. Therefore a simple brute-force algorithm can easily solve the active measurement selection problem. In graph pruning brute-force search has to check all of the $m$ candidate. On an Intel Core i5-2400 CPU, the time required to solve the graph pruning problem in City $10 \mathrm{~K}$ with $\mathcal{C}^{-}=\mathcal{E}$ (i.e., more than $2 \times 10^{4}$ edges) is less than 3 seconds using our simple MATLAB implementation. The active measurement selection problem with $\left|\mathcal{C}^{+}\right|=100$ candidates can be solved in less than 0.03 second on the same machine.

\section{Results}

Similar to what we did in Section III-B, here we created 100 random subgraphs (with fixed number of poses and edges) from the Intel Research Lab and Manhattan (with 4 different $\sigma_{\theta}$ ) datasets. In this experiment, we used the set of loop-closure edges as our candidate set $\mathcal{C}^{-}$. In each subgraph, we sorted the edges in $\mathcal{C}^{-}$according to our objective function (27), and specified the least valuable edge for pruning. We also ranked the edges in $\mathcal{C}^{-}$for each subgraph according to the true D-optimality criterion, and found the (true) least valuable edge by exhaustive search. In order to compare our ranking with the correct ranking, we use the Kendall's $\tau$ rank correlation coefficient [25]. For $\tau$ we have: $\tau \in[-1,1]$. Moreover, $\tau=1$ iff the two ranked lists are identical, and $\tau=-1$ iff one of the rankings is exactly the reverse of the other ranking.

Table I shows the results. The average of $\tau$ over 100 Monte Carlo simulations is denoted by $\bar{\tau}$. The standard errors are also computed for $\bar{\tau}$. As we expected (see Remark 5), there is a substantial positive correlation between our ranking and the true ranking, even when $\alpha$ is not large enough. Note that the correlation becomes stronger as $\sigma_{\theta}$ decreases. It is also clear that almost in all of the random subgraphs, our graph pruning strategy was able to prune the least valuable edge in terms of the D-optimality criterion.

\section{Discussion \& CONCLUSiON}

This paper is the first step towards revealing the important role played by the graph structure in the accuracy of ML estimate in SLAM. We started our analysis by discussing a heuristic measure proposed by Olson and Kaess [12]. We provided a proof for their empirical observation regarding the impact of average node degree on the reliability of the ML estimate. For a fixed number of poses, the average node degree is basically reflecting the number of measurements, and cannot distinguish between different distributions of edges.

Then we revealed the exact relation between the number of spanning trees and the determinant of the covariance matrix of MLE in compass-SLAM and linear-SN. According to our experiments, this result can be extended to the nonlinear SLAM problem if the orientation noise is relatively small. It is very important to note that even when the orientation noise is not sufficiently small, there is still a strong positive correlation between the number of spanning trees and the Doptimality criterion. We also revealed the relation between the algebraic connectivity and the largest eigenvalue of the covariance of MLE in compass-SLAM and linear-SN. Finally, we applied our results on active measurement selection and edge pruning. The outcome confirms the fact that the number of spanning trees is an important measure of connectivity and reliability in SLAM.

In order to reveal the impact of graph structure on different aspects of FIM, we need to isolate its effect from the effect of other factors, such as the accuracy of each measurement. Therefore it was deemed necessary to assume that all measurements have the same noise covariance matrix. It is worth noting that in fact the output of a considerable number of front-ends as well as many popular datasets are consistent with this assumption. Additionally, it is also possible to fuse the structural value of each edge with its information content (i.e., its noise covariance matrix) heuristically. In some applications, such as data gathering, it may be highly beneficial to avoid the expensive task of (exact) scan registration, and instead focus only on optimizing the quality of the graph structure. As an alternative strategy, one may prefer to first look at the potential structural value of a potential loopclosure, before deciding to perform the expensive task of scan registration.

Our analysis is useful in comparing the quality of two graphs (e.g., two datasets or planned trajectories) by just looking at the structure of the corresponding graphs (e.g., created by a simple appearance-based place recognition front-end) with a reasonable computational cost, without (or before) solving the actual SLAM problems. Ultimately our results can help us to collect reliable datasets in active scenarios. Decision making in active scenarios based on the graph structure has a major advantage over the existing 
TABLE I

GraPh PRUNing: 100 RANDOM SUBgRAPHS FOR EACH DATASET. HeRE $\bar{\tau}$ IS THE SAMPLE MEAN OF KENDALL'S $\tau$ RANK CORRELATION COEFFICIENT

[25]. OPTIMAL CHOICE SHOWS THE NUMBER OF TIMES OUR APPROACH (27) HAS REMOVED THE TRUE LEAST VALUABLE EDGE.

\begin{tabular}{|ccc||c|c||c|}
\hline Dataset & $\sigma_{p}(\mathrm{~m})$ & $\sigma_{\theta}(\mathrm{rad})$ & $\bar{\tau}$ & Standard Error & Optimal Choice (\%) \\
\hline Intel Research Lab (180 poses) & 0.0447 & 0.0141 & 0.9878 & 0.0010 & $92 \%$ \\
\hline Manhattan (200 poses) & 0.1495 & 0.2000 & 0.8998 & 0.0034 & $95 \%$ \\
\hline Manhattan (200 poses) & 0.1495 & 0.1495 & 0.9236 & 0.0019 & $95 \%$ \\
\hline Manhattan (200 poses) & 0.1495 & 0.1000 & 0.9491 & 0.0017 & $96 \%$ \\
\hline Manhattan (200 poses) & 0.1495 & 0.0500 & 0.9769 & 0.0009 & $99 \%$ \\
\hline
\end{tabular}

methods such as [17], in that the risk of converging to a local minimum or large linearization errors has no adverse impact on our decision. It is important to note that evaluating the Jacobian at an inaccurate estimate or a local minimum leads to an inconsistent estimate of covariance matrix.

For $\alpha \geq 1$ we can find lower and upper bounds for $\operatorname{det}(I+T)$ that depend on the noise variances, graph structure and the distances between the nodes. In future work we plan to improve our current bounds and investigate the relation between the graph structure and reliability of ML estimate in other SLAM formulations (e.g., 3D). We are also planning to use our results in practical problems in the context of sensor networks and active SLAM.

\section{APPENDIX I \\ PROOF OF LEMMA 1}

Proof. According to Theorem 2, the number of spanning trees in $\mathcal{G}^{\prime}$ is given by

$$
\mathrm{t}^{\prime}=\operatorname{det} L^{\prime}=\operatorname{det}\left(L+a^{i j} a^{i j^{\top}}\right) .
$$

Recall that $L \succ 0$ in SLAM. Therefore the rank-one update of $\operatorname{det}(L)$ can be written as [20, Chapter 6.2]

$$
\begin{aligned}
\mathrm{t}^{\prime} & =\operatorname{det}(L)\left(1+a^{i j^{\top}} L^{-1} a^{i j}\right) \\
& =\mathrm{t}\left(1+a^{i j^{\top}} L^{-1} a^{i j}\right) .
\end{aligned}
$$

Dividing both sides by $t$ completes the proof. Note that using the definition of $a^{i j}$ we can simplify $a^{i j} L^{-1} a^{i j}$ into

$$
a^{i j^{\top}} L^{-1} a^{i j}=\left(L^{-1}\right)_{i, i}+\left(L^{-1}\right)_{j, j}-2\left(L^{-1}\right)_{i, j} .
$$

\section{ACKNOWLEDGMENT}

The authors would like to thank the anonymous reviewers for their helpful suggestions and comments.

\section{REFERENCES}

[1] G. Dissanayake, P. Newman, S. Clark, H. Durrant-Whyte, and M. Csorba, "A solution to the simultaneous localization and map building (slam) problem," Robotics and Automation, IEEE Transactions on, vol. 17, no. 3, pp. 229-241, 2001.

[2] G. Grisetti, C. Stachniss, S. Grzonka, and W. Burgard, "A tree parameterization for efficiently computing maximum likelihood maps using gradient descent," in Proceedings of Robotics: Science and Systems, Atlanta, GA, USA, June 2007.

[3] M. Kaess, H. Johannsson, R. Roberts, V. Ila, J. J. Leonard, and F. Dellaert, "isam2: Incremental smoothing and mapping using the bayes tree," The International Journal of Robotics Research, vol. 31, no. 2, pp. 216-235, 2012.

[4] N. Sunderhauf and P. Protzel, "Towards a robust back-end for pose graph slam," in IEEE International Conference on Robotics and Automation (ICRA), 2012. IEEE, 2012, pp. 1254-1261.
[5] G. Hu, K. Khosoussi, and S. Huang, "Towards a reliable slam backend," in Intelligent Robots and Systems (IROS), 2013 IEEE/RSJ International Conference on, 2013.

[6] H. Wang, G. Hu, S. Huang, and G. Dissanayake, "On the structure of nonlinearities in pose graph SLAM," in Proceedings of Robotics: Science and Systems, Sydney, Australia, July 2012.

[7] L. Carlone, "Convergence analysis of pose graph optimization via gauss-newton methods," in Proceedings of the IEEE International Conference on Robotics and Automation (ICRA), May 2013.

[8] S. Huang and G. Dissanayake, "Convergence and consistency analysis for extended kalman filter based slam," Robotics, IEEE Transactions on, vol. 23, no. 5, pp. 1036-1049, 2007.

[9] G. P. Huang, A. I. Mourikis, and S. I. Roumeliotis, "Analysis and improvement of the consistency of extended kalman filter based slam," in IEEE International Conference on Robotics and Automation (ICRA), 2008. IEEE, 2008, pp. 473-479.

[10] F. Pukelsheim, Optimal design of experiments. SIAM, 1993, vol. 50.

[11] M. Fiedler, "Algebraic connectivity of graphs," Czechoslovak Mathematical Journal, vol. 23, no. 2, pp. 298-305, 1973.

[12] E. Olson and M. Kaess, "Evaluating the performance of map optimization algorithms," in RSS Workshop on Good Experimental Methodology in Robotics, 2009, p. 40.

[13] P. Barooah and J. P. Hespanha, "Estimation on graphs from relative measurements," Control Systems, IEEE, vol. 27, no. 4, pp. 57-74, 2007.

[14] L. Carlone, R. Aragues, J. Castellanos, and B. Bona, "A linear approximation for graph-based simultaneous localization and mapping," in Proceedings of Robotics: Science and Systems, Los Angeles, CA, USA, June 2011.

[15] H. Sorenson, Parameter estimation: principles and problems, ser. Control and systems theory. M. Dekker, 1980.

[16] W. H. Press, S. A. Teukolsky, W. T. Vetterling, and B. P. Flannery, Numerical recipes 3 rd edition: The art of scientific computing. Cambridge University Press, 2007.

[17] T. A. Vidal-Calleja, A. Sanfeliu, and J. Andrade-Cetto, "Action selection for single-camera slam," Systems, Man, and Cybernetics, Part B: Cybernetics, IEEE Transactions on, vol. 40, no. 6, pp. 1567-1581, 2010.

[18] A. J. Davison, "Active search for real-time vision," in Computer Vision, 2005. ICCV 2005. Tenth IEEE International Conference on, vol. 1. IEEE, 2005, pp. 66-73.

[19] C. Godsil and G. Royle, Algebraic graph theory, ser. Graduate Texts in Mathematics Series. Springer London, Limited, 2001.

[20] C. D. Meyer, Matrix analysis and applied linear algebra. Siam, 2000.

[21] Y. Kim and M. Mesbahi, "On maximizing the second smallest eigenvalue of a state-dependent graph laplacian," Automatic Control, IEEE Transactions on, vol. 51, no. 1, pp. 116-120, 2006.

[22] S. Joshi and S. Boyd, "Sensor selection via convex optimization," Signal Processing, IEEE Transactions on, vol. 57, no. 2, pp. 451-462, 2009.

[23] V. Ila, J. M. Porta, and J. Andrade-Cetto, "Information-based compact pose slam," Robotics, IEEE Transactions on, vol. 26, no. 1, pp. 78-93, 2010.

[24] Y. Chen, T. A. Davis, W. W. Hager, and S. Rajamanickam, "Algorithm 887: Cholmod, supernodal sparse cholesky factorization and update/downdate," ACM Transactions on Mathematical Software (TOMS), vol. 35, no. 3, p. 22, 2008.

[25] M. G. Kendall, "A new measure of rank correlation," Biometrika, vol. 30, no. 1/2, pp. pp. 81-93, 1938. [Online]. Available: http://www.jstor.org/stable/2332226 